1 Hacettepe Journal of Mathematics and Statistics

$\bigcap$ Volume 45 (5) (2016), 1421-1434

\title{
Quasi-primry submodules satisfying the primeful property I
}

\author{
Hosein Fazaeli Moghimi ${ }^{* \dagger}$ and Mahdi Samiei ${ }^{\ddagger}$
}

\begin{abstract}
Let $R$ be a commutative ring with identity and $M$ a unital $R$-module. In this article we extend the notion of quasi-primary ideals to submodules. A proper submodule $N$ of $M$ is called quasi-primary if whenever $r x \in N$ for $r \in R$ and $x \in M$, then $r \in \sqrt{(N: M)}$ or $x \in \operatorname{rad} N$ where $\operatorname{rad} N$ is the intersection of all prime submodules of $M$ containing $N$. Also, we say that a submodule $N$ of $M$ satisfies the primeful property if $M / N$ is a primeful $R$-module. For a quasi-primary submodule $N$ of $M$ satisfying the primeful property, $\sqrt{(N: M)}$ is a prime ideal of $R$. For the existence of a module-reduced quasi-primary decomposition, the radical of each term appeared in decomposition must be prime. We provide sufficient conditions, involving the saturation and torsion arguments, to ensure that this property holds as is valid in the ideal case. It is proved that for a submodule $N$ of $M$ over a Dedekind domain $R$ which satisfies the primeful property, $N$ is quasi-primary if and only if $\operatorname{rad} N$ is prime.
\end{abstract}

Keywords: Quasi-primary submodule, Primeful property, Prime submodule, Radical of a submodule, Saturation, Torsion.

2000 AMS Classification: 13C13, 13C99

Received : 26.01.2013 Accepted: 02.12.2015 Doi : 10.15672/HJMS.20164514283

*Department of Mathematics, University of Birjand, P.O.Box 97175-615, Birjand, Iran, Email: hfazaeli@birjand.ac.ir

${ }^{\dagger}$ Corresponding Author.

${ }^{\ddagger}$ Department of Mathematics, University of Birjand, P.O.Box 97175-615, Birjand, Iran, Email: mahdisamiei@birjand.ac.ir 


\section{Introduction}

Throughout this paper all rings are commutative with non-zero identity and all modules are unital. If $R$ is a ring and $N$ a submodule of an $R$-module $M$, the ideal $\{r \in R \mid r M \subseteq N\}$ will be denoted by $(N: M)$. Then ann $(M)$, the annihilator of $M$, is $(0: M)$. A proper submodule $N$ of $M$ is said to be prime (resp. primary), if $r x \in N$ for $r \in R$ and $x \in M$ implies that either $r \in(N: M)$ (resp. $r \in \sqrt{(N: M)}$ ) or $x \in N$. In this case, $N$ is called $p$-prime (resp. $p$-primary), where $p=(N: M)$ (resp. $p=\sqrt{(N: M)}$ ) (For more study these notions see for example $[3,13,14,16,17,19]$ ). The intersection of all prime submodules containing $N$, denoted $\operatorname{rad} N$, is called the prime radical of $N$. Also, $N$ is called a radical submodule if $\operatorname{rad} N=N$. A proper submodule $N$ of $M$ is called primary-like if $r x \in N$ for $r \in R$ and $x \in M$ implies that $r \in(N: M)$ or $x \in \operatorname{radN}$. It is clear that primary-like submodules of $R$ as an $R$-module and primary ideals of $R$ are the same. Also, $N$ is a prime submodule of $M$ if and only if $N$ is a radical and primary-like submodule of $M$. The notion of primary-like submodules has been extensively studied by the authors and F. Rashedi in [6].

A proper ideal $q$ of $R$ is said to be quasi-primary if $r s \in q$ for $r, s \in R$ implies $r \in \sqrt{q}$ or $s \in \sqrt{q}$. In particular, $q$ is a quasi-primary ideal of $R$ if and only if $\sqrt{q}$ is a prime ideal of $R$ [7, p.176]. Quasi-primary ideals was first introduced and studied by L. Fuchs [7]. Since primary ideals are quasi-primary, every ideal of a Noetherian ring has a quasi-primary decomposition. Moreover, the uniqueness of the corresponding shortest quasi-primary decompositions of an ideal has been given in [7, Theorem 6]. Here we extend the notion of quasi-primary ideals to submodules. Recall that a proper submodule $N$ of $M$ is quasi-primary if $r x \in N$ for $r \in R$ and $x \in M$ implies that $r \in \sqrt{(N: M)}$ or $x \in \operatorname{radN}$. It is clear that primary submodules are quasi-primary. We say that a submodule $N$ of an $R$-module $M$ satisfies the primeful property if for each prime ideal $p$ of $R$ with $(N: M) \subseteq p$, there exists a prime submodule $P$ containing $N$ such that $(P: M)=p$. If the zero submodule of $M$ satisfies the primeful property, then $M$ is called primeful. For instance finitely generated modules, projective modules over domains and (finite and infinite dimensional) vector spaces are primeful (see [10]). If $N$ satisfies the primeful property, then $\sqrt{(N: M)}=(\operatorname{rad} N: M)$ [10, Proposition 5.3]. If $N$ is a quasi-primary (primary-like) submodule satisfying the primeful property, then it is easy to verify that $p=\sqrt{(N: M)}$ is a prime ideal of $R$. In this case, $N$ is called a $p$-quasi-primary $(p-$ primary like) submodule of $M$. In [4], Atani and Darani used the term "quasi-primary submodule" in a different way. In fact, they consider a submodule $N$ of an $R$-module $M$ as a quasi-primary submodule if $\sqrt{(N: M)}$ is a prime ideal of $R$. Thus a quasi-primary submodule satisfying the primeful property, in the our sense, follows that in [4]. But the converse is not true in general. For example, if $M=\prod_{p \in \Omega} \mathbb{Z} / p \mathbb{Z}$ and $N=\bigoplus_{p \in \Omega} \mathbb{Z} / p \mathbb{Z}$ are $\mathbb{Z}$-modules, where $\Omega$ is the set of prime integers, then $M$ is a primeful module and $N$ is a 0 -prime submodule of $M$ with $\operatorname{rad}(N)=0$ while $N$ dose not satisfy the primeful property, i.e. $M / N$ is not primeful [10, Example 1 (5)]. Now we give an example of a submodule $N$ such that $\sqrt{(N: M)}$ is a prime ideal while $N$ is not quasi-primary. Consider the $\mathbb{Z}$-module $M=\mathbb{Q} \oplus \mathbb{Z}_{p}$, where $\mathbb{Q}$ is the additive abelian group of rational numbers and $\mathbb{Z}_{p}$ is the cyclic group of order $p$. Then $\mathbb{Q} \oplus 0$ and $0 \oplus \mathbb{Z}_{p}$ are only prime submodules of $M$ [14, Example 2.6]. Now if $N=0 \oplus 0$, it is easy to verify that $(N: M)=0$ and $N$ is not a quasi-primary submodule of $M$. Also, a quasi-primary submodule does not satisfy the primeful property necessarily. For example, if $M=\mathbb{Z}\left(p^{\infty}\right) \oplus \mathbb{Z}_{p}$ as a $\mathbb{Z}$-module, where $\mathbb{Z}\left(p^{\infty}\right)$ is the Prüfer group, and $N=0 \oplus \mathbb{Z}_{p}$, then $\operatorname{rad} N=M$ and so $N$ is a quasi-primary submodule of $M$. But $N$ dose not satisfy the primeful property [14, Example 3.7].

We say that a submodule $N$ of an $R$-module $M$ has a quasi-primary decomposition if $N=N_{1} \cap N_{2} \cap \cdots \cap N_{t}$, where each $N_{i}$ is a quasi-primary submodule of $M$. If 
$N_{i} \nsupseteq N_{1} \cap \cdots \cap N_{i-1} \cap N_{i+1} \cap \cdots \cap N_{t}$, for $1 \leq i \leq t$, then the above quasi-primary decomposition is called (1) a reduced quasi-primary decomposition, if the ideals $\sqrt{\left(N_{i}: M\right)}$ are distinct primes; (2) a module-reduced quasi-primary decomposition, if the submodules $\operatorname{radN}_{i}$ are distinct primes; (3) a shortest quasi-primary decomposition, if none of the intersection $\left(N_{i_{1}}: M\right) \cap\left(N_{i_{2}}: M\right) \cap \cdots \cap\left(N_{i_{s}}: M\right)(s>1)$ is a quasi-primary ideal. In part II, we investigate the existence and uniqueness of these decompositions and relationships between them in different cases. For this purpose we need to some properties and facts about quasi-primary submodules, mostly consideration satisfying the primeful property.

Unlike the ideal case, there are several challenging problems in radical theory of submodules. Finding a good description of $\operatorname{radN}$ either in terms of its elements or as some sort of decomposition and splitting the finite intersection of submodules by radical are two examples of them. Some works and methods for characterizing the $\operatorname{radN}$ may be found in $[1,12,15,16,18,19,20,21])$. One of the main differences between ideal and module cases is that the radical of a quasi-primary submodule is not necessarily prime. In fact, if $R=\mathbb{Z}[x]$, then the submodule $N=R(2, x)+R(x, 0)$ is a quasi-primary submodule of $M=R \oplus R$ whose radical is not prime [19, Theorem 1.9 and Example 1.11]. The mentioned conditions are useful to obtain a module-reduced quasi-primary decomposition from the original one.

In section 2, the behaviuor of quasi-primary submodules (probably satisfying the primeful property) under some operations such as quotient and fraction are considered (Corollary ?? and Theorem 2.14). In this section, it is also shown that $q$ is a quasiprimary ideal of $R$ if and only if $q F$ is a quasi-primary submodule of a free $R$-module $F$ (Theorem 2.18). In this case $\operatorname{rad}(q F)$ is a prime submodule of $F$. Moreover, it is proved that the radical of every quasi-primary submodule of a free module $F$ over a Noetherian domain $R$ is prime provided that every prime submodule of $F$ contains only finitely many prime submodules (Proposition 2.20).

Let $p$ be a prime ideal of $R$ and $N$ a submodule of $M$. By the saturation of $N$ with respect to $p$, we mean the contraction of $N_{p}$ in $M$ and designate it by $S_{p}(N)$. It is also known that $S_{p}(N)=\{x \in M \mid c x \in N$ for some $c \in R \backslash p\}$. Saturations of submodules were investigated in detail in [11] and some results of the study are applied for quasi-primary submodules in section 3. For example, if $N$ is a $p$-quasi-primary submodule satisfying the primeful property, then we have : (1) $S_{p}(N)$ is a prime submodule of $M$ if and only if $\operatorname{rad} N=S_{p}(N)$ (Theorem 3.3); (2) $S_{p}(\operatorname{rad} N) \neq M$ if and only if $\operatorname{rad} N$ is a prime submodule of $M$ (Theorem 3.9). Also, some other conditions under which the radical of a quasi-primary (probably satisfying the primeful property) is prime have been given in Corollary 3.7, Proposition 3.10, Corollary 3.11 and Theorem 3.14.

The purpose of the section 4 is to discuss about important roles played by torsion submodules in the class of quasi-primary submodules of a module. In Theorem 4.3, it is proved that for a submodule $N$ of a module $M$ over a Dedekind domain $R$ satisfying the primeful property, $\operatorname{rad} N$ is prime if and only if $M=\operatorname{rad} N \oplus N^{\prime}$ for some torsion-free submodule $N^{\prime}$ of $M$ or $(\operatorname{rad} N: M)=m$ for some maximal ideal $m$ of $R$.

In part II, we will characterize the quasi-primary submodules of multiplication modules. Using this, we will fully investigate reduced and module-reduced and shortest quasi-primary decompositions of submodules of multiplication modules. Also, we will give some uniqueness theorems for reduced and module-reduced quasi-primary decompositions of submodules of modules over Noetherian rings. 


\section{On quasi-primary submodules satisfying the primeful property}

In this section, we study basic properties of quasi-primary submodules which probably satisfies the primeful property. In particular we show the affect of some operations on quasi-primary submodules. We start with some elementary results.

2.1. Lemma. Let $M$ be an R-module. Then the following hold:

(i) Any maximal, prime, primary and primary-like submodule is quasi-primary.

(ii) Any quasi-primary radical submodule is primary. In particular, if $\operatorname{radN}$ is a quasi-primary submodule for a submodule $N$ of $M$, then rad $N$ is primary.

(iii) If $N$ is a quasi-primary submodule of $M$ and $(N: M)$ is a radical ideal of $R$, then $N$ is primary-like.

2.2. Lemma. Let $M$ be an $R$-module. If $N$ is a quasi-primary submodule of $M$ satisfying the primeful property with $p=\sqrt{(N: M)}$, then $\operatorname{rad} N=\operatorname{rad}(N+p M)$.

Proof. Clearly $\operatorname{rad} N \subseteq \operatorname{rad}(N+p M)$. If $P_{i}$ is a $p_{i}$-prime submodule such that $N \subseteq P_{i}$, then $p=\sqrt{(N: M)}=(\operatorname{rad} N: M) \subseteq\left(P_{i}: M\right)=p_{i}$. Hence $N+p M \subseteq P_{i}+p_{i} M \subseteq P_{i}$. Therefore $\operatorname{rad}(N+p M) \subseteq \operatorname{rad} N$.

2.3. Theorem. Let $m$ be a maximal ideal of $R$ and $M$ an $R$-module. If $N$ is an $m$ quasi-primary submodule of $M$ satisfying the primeful property, then radN is an m-prime submodule of $M$. Moreover, $\operatorname{rad} N=\operatorname{rad}(N+m M)=N+m M$.

Proof. Since $N$ satisfies the primeful property, we have $(\operatorname{rad} N: M)=\sqrt{(N: M)}=m$ and so $\operatorname{rad} N$ is an $m$-prime submodule of $M$. By Lemma 2.2, $N+m M \subseteq \operatorname{rad}(N+m M)=$ $\operatorname{rad} N$. Sine $\operatorname{rad} N$ is $m$-prime, we conclude $m \subseteq(N+m M: M) \subseteq(\operatorname{rad} N: M)=m$. It follows that $(N+m M: M)=m$. Hence $N+m M$ is a prime submodule containing $N$. Thus $\operatorname{rad} N=\operatorname{rad}(N+m M)=N+m M$.

2.4. Proposition. Let $M$ be an $R$-module. If $N$ is a quasi-primary submodule of $M$ and $L$ a submodule of $M$ such that $\operatorname{rad} N \cap \operatorname{rad} L=\operatorname{rad}(N \cap L)$, then $L \subseteq N$ or $N \cap L$ is a quasi-primary submodule of $L$.

Proof. Suppose $L \nsubseteq N$. Let $r l \in N \cap L$ for $r \in R \backslash \sqrt{(N \cap L: L)}$ and $l \in L$. Then $r l \in N$ and $r \notin \sqrt{(N: M)}$. Since $N$ is a quasi-primary submodule of $M$, we have $l \in \operatorname{rad} N$. Thus $l \in \operatorname{rad} N \cap \operatorname{rad} L=\operatorname{rad}(N \cap L)$.

2.5. Corollary. Let $N$ and $K$ be proper submodules of an $R$-module $M$. If $N$ is a quasiprimary submodule of $M$ satisfying the primeful property such that $N \varsubsetneqq K$, then $N$ is also a quasi-primary submodule of $K$.

Proof. It follows by applying Proposition 2.4 to $N$ and $K$.

2.6. Theorem. Let $N$ be a proper submodule of a non-zero $R$-module $M$. Then the following statements are equivalent:

(i) $N$ is a quasi-primary submodule of $M$;

(ii) $\sqrt{(N: K)}=\sqrt{(N: M)}$ for every submodule $K$ of $M$ such that $K \supseteq \operatorname{rad} N$.

Proof. (i) $\Rightarrow$ (ii). Let $K$ be any submodule of $M$ such that $K \nsupseteq \operatorname{rad} N$. Then $K / N \subseteq M / N$ and so, $\sqrt{(N: K)} \supseteq \sqrt{(N: M)}$. For the reverse inclusion, let $a \in \sqrt{(N: K)}$. Since $\operatorname{rad} N \varsubsetneqq K$, we can find an element $x$ of $K \backslash \operatorname{rad} N$. Then $a^{n} x \in N$ for some positive integer $n$. Hence, by (i), $a \in \sqrt{(N: M)}$.

(ii) $\Rightarrow$ (i). Suppose $r x \in N$, where $r \in R$ and $x \in M$. Assume $x \notin \operatorname{rad} N$. Then $\operatorname{rad} N \subsetneq$ $\operatorname{rad} N+R x \subseteq M$. By (ii), $\sqrt{(N: \operatorname{rad} N+R x)}=\sqrt{(N: M)}$. Since $r x \in N$, we have 
$r(N+R x)=r N+R r x \subseteq N$. This shows that $r \in(N: N+R x) \subseteq \sqrt{(\operatorname{rad} N: N+R x)}$. Hence $r \in \sqrt{(N: M)}$, as required.

2.7. Theorem. Let $\left\{N_{i}: 1 \leq i \leq n\right\}$ be a finite collection of submodules of an $R$-module $M$ satisfying the primeful property. Then $\cap_{i=1}^{n} N_{i}$ satisfies the primeful property and $\sqrt{\left(\cap_{i=1}^{n} N_{i}: M\right)}=\left(\operatorname{rad}\left(\cap_{i=1}^{n} N_{i}\right): M\right)$.

Proof. Suppose $p$ is a prime ideal of $R$ containing $\left(\cap_{i=1}^{n} N_{i}: M\right)$. Then $\left(N_{j}: M\right) \subseteq p$, for some $1 \leq j \leq n$. Since $N_{j}$ satisfies the primeful property, there exists a prime submodule $P$ of $M$ containing $N_{j}$ with $(P: M)=p$. Hence $\cap_{i=1}^{n} N_{i}$ satisfies the primeful property and so $\sqrt{\left(\cap_{i=1}^{n} N_{i}: M\right)}=\left(\operatorname{rad}\left(\cap_{i=1}^{n} N_{i}\right): M\right)$.

The following is a result of Theorem 2.7.

2.8. Corollary. Let $M$ be an $R$-module and $\left\{N_{i}: i \in I\right\}$ a collection of quasi-primary submodules of $M$ satisfying the primeful property. Then $\left(\operatorname{rad}\left(\cap_{i=1}^{n} N_{i}\right): M\right)=\left(\cap_{i=1}^{n} \operatorname{rad} N_{i}\right.$ : $M)$.

It is well-known that for a surjective homomorphism $f: M \rightarrow M^{\prime}$ and a prime submodule $N$ of $M$ containing $\operatorname{Ker} f, f(N)$ is a prime submodule of $M^{\prime}$. It follows that for any submodule $N$ of $M, f(\operatorname{rad} N) \subseteq \operatorname{rad} f(N)$. Also if $\operatorname{Ker} f \subseteq N$, then $f(\operatorname{rad} N)=\operatorname{rad} f(N)$. In particular for every submodule $K$ of $M$ containing $N, \operatorname{rad}(K / N)=\operatorname{rad} K / N$. Analogously we have the following corollaries:

2.9. Theorem. Let $f: M \rightarrow M^{\prime}$ be a surjective homomorphism. If $N^{\prime}$ is a quasiprimary submodule of $M^{\prime}$ such that $f^{-1}\left(N^{\prime}\right)$ is containing Kerf, then $f^{-1}\left(N^{\prime}\right)$ is a quasi-primary submodule of $M$.

Proof. Suppose $r m^{\prime} \in f^{-1}\left(N^{\prime}\right)$ and $r \notin \sqrt{\left(f^{-1}\left(N^{\prime}\right): M\right)}$. It follows that $r f\left(m^{\prime}\right) \in N^{\prime}$ and $r \notin \sqrt{\left(N^{\prime}: M^{\prime}\right)}$. Since $N^{\prime}$ is a quasi-primary submodule of $M^{\prime}, f\left(m^{\prime}\right) \in \operatorname{rad} N^{\prime}$; i.e. $f\left(m^{\prime}\right) \in P^{\prime}$ for any prime submodule $P^{\prime}$ of $M^{\prime}$ containing $N^{\prime}$. Now, let $P$ be a prime submodule of $M$ containing $f^{-1}\left(N^{\prime}\right)$. Then $N^{\prime}=f f^{-1}\left(N^{\prime}\right) \subseteq P$. Since $f(P)$ is a prime submodule of $M^{\prime}$ containing $N^{\prime}$, we must have $f\left(m^{\prime}\right) \in f(P)$. Therefore, there exists an element $x \in P$ such that $m^{\prime}-x \in \operatorname{Ker} f \subseteq P$. Thus $m^{\prime} \in P$ and so $m^{\prime} \in \operatorname{rad}\left(f^{-1}\left(N^{\prime}\right)\right)$.

2.10. Theorem. Let $f: M \rightarrow M^{\prime}$ be a surjective homomorphism and $N$ a submodule of $M$. If $N$ is a quasi-primary submodule of $M$ containing $\operatorname{Ker} f$, then $f(N)$ is a quasiprimary submodule of $M^{\prime}$.

Proof. Suppose that $r f(x) \in f(N)$ for $r \in R$ and $x \in M$ and $r \notin \sqrt{(f(N): f(M))}$. Hence there exists $n \in N$ such that $r x-n \in \operatorname{Ker} f$. Therefor $r x \in N$ and so we have $x \in \operatorname{rad} N$. Since $f(\operatorname{rad} N)=\operatorname{rad}(f(N))$, we conclude that $f(x) \in \operatorname{rad}(f(N))$.

2.11. Corollary. Let $f: M \rightarrow M^{\prime}$ be a surjective homomorphism. Then the assignment $N \mapsto f(N)$ defines a one-to-one correspondence between the set of all quasi-primary submodules of $M$ containing Kerf and the set of all quasi-primary submodules $N^{\prime}$ of $M^{\prime}$ such that $f^{-1}\left(N^{\prime}\right)$ contains Kerf.

From now on, we frequently use the fact that $(\operatorname{rad} N: M)=\sqrt{(N: M)}$ for a submodule $N$ of $M$ which satisfies the primeful property. Specially it is used in items (ii) and (iii) of the following immediate results.

2.12. Lemma. Let $N$ be a submodule of an $R$-module $M$ satisfying the primeful property. Then the following hold: 
(i) If $N$ is a quasi-primary submodule of $M$, then $(N: M)$ is a quasi-primary ideal of $R$.

(ii) $\operatorname{radN}$ is quasi-primary if and only if radN is primary-like if and only if radN is primary if and only if radN is prime.

(iii) If radN is a prime submodule of $M$, then $N$ is quasi-primary.

2.13. Theorem. Let $N$ be a proper submodule of a finitely generated module $M$ over a zero-dimensional ring $R$. Then $N$ is quasi-primary if and only if there exists a quasiprimary ideal $q$ of $R$ such that $q \subseteq(N: M)$. In particular, $N$ is a quasi-primary submodule of $M$ if and only if $(N: M)$ is a quasi-primary ideal of $R$.

Proof. Since $M$ is finitely generated, $N$ satisfies the primeful property, then Lemma 2.12 follows that $(N: M)$ is a quasi-primary ideal. Conversely, let $q$ be a quasi-primary ideal of $R$ such that $q \subseteq(N: M)$. Since $M$ is finitely generated, $N$ is contained in a maximal submodule of $M$ and so $\operatorname{rad} N \neq M$. Since $R$ is zero-dimensional, $\sqrt{q}$ is a maximal ideal of $R$ and so $\sqrt{q}=\sqrt{(N: M)}=(\operatorname{rad} N: M)$. Hence $\operatorname{rad} N$ is a prime submodule of $M$. Therefore by Lemma 2.12 (iii), $N$ is quasi-primary.

Let $S$ be a multiplicatively closed subset of $R$ and $M$ an $R$-module. We denote the ring and module of fractions by $S^{-1} R$ and $S^{-1} M$ respectively.

2.14. Theorem. Let $M$ be an $R$-module and $N$ a quasi-primary submodule of $M$ satisfying the primeful property. Let $S$ be a multiplicatively closed subset of $R$ such that $S \cap \sqrt{(N: M)}=\emptyset$. Then $S^{-1} N$ is a quasi-primary submodule of $S^{-1} R$-submodule $S^{-1} M$.

Proof. It is easy to see that $x / 1 \in S^{-1} M \backslash S^{-1} N$ for each $x \in M \backslash \operatorname{rad} N$ and so $S^{-1} N \neq$ $S^{-1} M$. Suppose $(r / s)(x / t) \in S^{-1} N$ and $r / s \notin \sqrt{\left(S^{-1} N: S^{-1} M\right)}$. Since $S^{-1} \sqrt{(N: M)} \subseteq$ $\sqrt{\left(S^{-1} N: S^{-1} M\right)}$, then $r \notin \sqrt{(N: M)}$. Thus there exist $u, w \in S, y \in N$ such that wur $x=$ wsty. It follows $x \in \operatorname{rad} N$, since $N$ is quasi-primary. Thus $x / t \in S^{-1} \mathrm{rad} N \subseteq$ $\operatorname{rad}\left(S^{-1} N\right)$, by [16, Theorems 3.3 and Theorem 3.4].

In the following the localization of a ring $R$ and an $R$-module $M$ at a prime ideal $p$ are denoted by $R_{p}$ and $M_{p}$ respectively.

2.15. Theorem. Let $M$ be an $R$-module and $N$ a quasi-primary submodule of $M$ satisfying the primeful property. Then $(\operatorname{radN})_{p}$ is an $R_{p}$-prime submodule of $M_{p}$ where $p=\sqrt{(N: M)}=(\operatorname{rad} N: M)$. In addition, $\operatorname{rad} N_{p}$ is prime and $\operatorname{rad} N_{p}=(\operatorname{rad} N)_{p}$.

Proof. By [16, Theorems 3.3 and Theorem 3.4] $(\operatorname{radN})_{p} \subseteq \operatorname{rad}\left(N_{p}\right)$. For the reverse inclusion, it is easy to see that $(\operatorname{rad} N: M)_{p} \subseteq\left((\operatorname{rad} N)_{p}: M_{p}\right)$. Since $N$ is quasiprimary, by Lemma $2.12(\mathrm{i}),(\operatorname{rad} N: M)_{p}$ is the unique maximal ideal of $R_{p}$. Now we have $(\operatorname{rad} N: M)_{p}=\left((\operatorname{rad} N)_{p}: M_{p}\right)$, because $(\operatorname{rad} N)_{p} \neq M_{p}$. Thus $(\operatorname{rad} N)_{p}$ is a prime submodule of $M_{p}$ containing $N_{p}$. On the other hand, by [18, Lemma 1.7] $\operatorname{rad}\left(N_{p}\right)$ is a prime submodule of $M_{p}$ containing $N_{p}$. Hence $\operatorname{rad}\left(N_{p}\right) \subseteq(\operatorname{rad} N)_{p}$.

We remark that if $N$ is a submodule of $M$ satisfying the primeful property, then $\operatorname{rad} N$ is also satisfies the primeful property. In this case if $N$ is a proper submodule of $M$, then $\operatorname{rad} N$ is also proper. Henceforth, we consider $\operatorname{rad} N \neq M$ when trying to prove $\operatorname{rad} N$ is prime for a quasi-primary submodule $N$ satisfying the primeful property.

2.16. Proposition. Let $R$ be a ring and $N$ a quasi-primary submodule of an $R$-module $M$ satisfying the primeful property. If $\sqrt{(N: M)}$ is a maximal ideal of $R$, then radN is a prime submodule of $M$. 
2.17. Proposition. Let $M$ be an R-module and $\left\{N_{i}: i \in I\right\}$ a collection of submodules of $M$ such that $\sum_{i \in I} N_{i}$ satisfies the primeful property. Then $\sum_{i \in I} \operatorname{radN} N_{i}=M$ if and only if $\sum_{i \in I} N_{i}=M$.

Proof. Assume $\sum_{i \in I} \operatorname{rad} N_{i}=M$ and $\sum_{i \in I} N_{i} \neq M$. Then there exists a maximal ideal $m$ of $R$ containing $\left(\sum_{i \in I} N_{i}: M\right)$ and a prime submodule $P$ of $M$ containing $\sum_{i \in I} N_{i}$ such that $(P: M)=m$. Thus $\sum_{i \in I} \operatorname{rad} N_{i} \subseteq P$, a contradiction. The converse is obvious.

It is well-known that if $F$ is a free $R$-module and $I$ is an ideal of $R$, then $(I F: F)=I$ and $\operatorname{rad}(I F)=\sqrt{I} F$ [20, Proposition 2.2]. Thus if $I$ is a prime(resp. primary) ideal of $R$, then $I F$ is prime(resp. primary) submodule of $M$. Now we give a similar result in the quasi-primary case.

2.18. Theorem. Let $F$ be a free $R$-module. Then $q F$ is a quasi-primary submodule of $F$ if and only if $q$ is a quasi-primary ideal of $R$.

Proof. Let $q F$ be a quasi-primary submodule of $M$. Since $(q F: F)=q, q$ is a proper ideal of $R$. Suppose $r s \in q$, for $r \in R, s \in R \backslash \sqrt{q}$. Hence $r s F \subseteq q F$ and $s \notin(\operatorname{radqF}: F)$, since $\operatorname{radq} F=\sqrt{q} F$ [20, Proposition 2.2]. It follows that $r \in \sqrt{q F: F}=\sqrt{q}$. Conversely let $q$ be a quasi-primary ideal of $R$. Again by $(q F: F)=q, q F$ is a proper submodule of $F$. Suppose $r \notin \sqrt{(q F: F)}=\sqrt{q}$ and $x \notin \operatorname{radqF}=\sqrt{q} F$. Hence we have $r x \notin \sqrt{q} F$, since $\sqrt{q} F$ is a prime submodule of $F$. Thus $r x \notin q F$.

2.19. Corollary. Let $F$ be a free $R$-module. Then the following statements are equivalent.

(i) $I=q_{1} \cap \cdots \cap q_{t}$ is a reduced quasi-primary decomposition of the ideal I;

(ii) $I F=q_{1} F \cap \cdots \cap q_{t} F$ is a reduced quasi-primary decomposition of IF;

(iii) $I F=q_{1} F \cap \cdots \cap q_{t} F$ is a module-reduced quasi-primary decomposition of IF.

2.20. Proposition. If $R$ is a Noetherian domain and $F$ is a free $R$-module such that every prime submodule of $F$ contains only finitely many prime submodules, then for every non-zero quasi-primary submodule $N$ of $F$, radN is prime.

Proof. We first show that $R$ is a one-dimensional ring. Let $0 \subset p^{\prime} \subseteq p$ be a chain of prime ideals of $R$. If $p^{\prime} \neq p$, then there exist infinitely many such prime ideals contained in $p[9$, p. 144]. It follows from the above argument of Theorem 2.18 that there exist infinitely many prime submodule contained in prime submodule $p F$, a contradiction. Thus $R$ is a one-dimensional domain. Now, let $q F$ be a non-zero quasi-primary submodule of $F$. It is clear that $0 \subset q \subseteq \sqrt{(q F: F)}$ and so the proof is completed by Proposition 2.16.

2.21. Theorem. Let $M$ be an R-module and $N$ a proper submodule of $M$. If $N_{1}, \cdots, N_{t}$ satisfies the primeful property and $N$ has a reduced quasi-primary decomposition $N=$ $N_{1} \cap N_{2} \cap \cdots \cap N_{t}$ such that all the prime ideals associated with $N$ are isolated, then $(N: M)=\left(N_{1}: M\right) \cap\left(N_{2}: M\right) \cap \cdots \cap\left(N_{t}: M\right)$ is a reduced quasi-primary decomposition of the ideal $(N: M)$ in $R$.

Proof. Suppose not. Since the ideals $\sqrt{\left(N_{i}: M\right)}$ are distinct, we have $\left(N_{i}: M\right) \supseteq$ $\cap_{j \neq i}\left(N_{j}: M\right)$ for some $i$. Then $\sqrt{\left(N_{i}: M\right)} \supseteq \cap_{j \neq i} \sqrt{\left(N_{j}: M\right)}$. It implies that $\sqrt{\left(N_{i}: M\right)} \supset$ $\sqrt{\left(N_{j}: M\right)}$ for some $i \neq j$, since $\sqrt{\left(N_{i}: M\right)}$ is a prime ideal. The final inclusion contradicts the assumption that $\sqrt{\left(N_{i}: M\right)}$ is an isolated prime ideal of $R$.

2.22. Corollary. Let $M$ be an R-module and $N$ a proper submodule of $M$. If $N_{1}, \cdots, N_{t}$ satisfies the primeful property and $N$ has a reduced quasi-primary decomposition $N=$ $N_{1} \cap N_{2} \cap \cdots \cap N_{t}$ such that all the prime ideals associated with $N$ are isolated, then 
(i) $N$ is quasi-primary if and only if $(N: M)$ is quasi-primary.

(ii) $N$ is prime if and only if $(N: M)$ is prime.

Proof. The necessity of each part is clear. To show sufficiency, let $N=N_{1} \cap N_{2} \cap \cdots \cap N_{t}$ be a reduce quasi-primary decomposition of $N$. By Theorem $2.21(N: M)=\left(N_{1}\right.$ : $M) \cap\left(N_{2}: M\right) \cap \cdots \cap\left(N_{t}: M\right)$ is a reduced quasi-primary decomposition of the ideal $(N: M)$ in $R$. If $(N: M)$ is quasi-primary, we must have $t=1$ and so $N=N_{1}$ is quasi-primary. (ii) is concluded by an analogous argument.

\section{Saturation and radical}

Let $p$ be a prime ideal of $R$ and $N$ a submodule of an $R$-module $M$. Then $S_{p}(N)=$ $\{x \in M: c x \in N$ for some $c \in R \backslash p\}$ is a submodule of $M$ which is called the saturation of $N$ with respect to $p$. A submodule $N$ of $M$ is called saturated with respect to $p$ if $S_{p}(N)=N$. It is easy to verify $S_{p}(N)$ is a saturated submodule of $M$ with respect to $p$. In [11], Lu applied the tool of saturation in the context of prime and primary submodules. In this section we develop and use this tool for quasi-primary submodules (probably satisfying the primeful property). In particular, using this, we give some conditions under which the radical of a quasi-primary submodule is prime.

3.1. Lemma. Let $N$ be a submodule of an R-module $M$ satisfying the primeful property. $N$ is a p-quasi-primary submodule of $M$ if and only if $\sqrt{(N: M)}=p$ is a prime ideal of $R$ and $S_{p}(N) \subseteq \operatorname{rad} N$.

Proof. Suppose $N$ is a $p$-quasi-primary submodule of $M$. Since $N$ satisfies the primeful property, it is clear that $\sqrt{(N: M)}=p$ is a prime ideal of $R$. Let $x \in S_{p}(N)$. Then $s x \in N$ for some $s \in R \backslash p$. Hence $x \in \operatorname{rad} N$ and so that $S_{p}(N) \subseteq \operatorname{rad} N$.

Assume $\sqrt{(N: M)}=p$ is a prime ideal of $R$. Let $r x \in N$ and $x \notin \operatorname{rad} N$. Hence we conclude that $s x \notin N$ for any $s \in R \backslash p$. Thus $r \in p$, as required.

From now on, we denote the set of all prime ideals of $R$ containing $(N: M)$ by $V(N: M)$.

3.2. Lemma. Let $N$ be a quasi-primary submodule of an $R$-module $M$. Then $S_{p}(N) \subseteq$ radN for every $p \in V(N: M)$. In particular, if $S_{p}(N)$ is a prime submodule of $M$ for some $p \in V(N: M)$, then $S_{p}(N)=\operatorname{rad} N$.

Proof. Straightforward.

3.3. Theorem. Let $N$ be a p-quasi-primary submodule of an $R$-module $M$ satisfying the primeful property. $S_{p}(N)$ is a p-prime submodule of $M$ if and only if $S_{p}(N)=\operatorname{rad} N$.

Proof. Assume that $S_{p}(N)$ is a $p$-prime submodule of $M$. It follows from Lemma 3.2 that $S_{p}(N)=\operatorname{rad} N$. Conversely, suppose $S_{p}(N)=\operatorname{rad} N$. Let $r x \in S_{p}(N)$ and $x \notin S_{p}(N)$. Then $r x \in N$ for some $r \in R \backslash p$. Since $N$ is a $p$-quasi-primary submodule of $M$, $r \in \sqrt{(N: M)}=(\operatorname{rad} N: M)=\left(S_{p}(N): M\right)$. Thus $S_{p}(N)$ is a $p$-prime submodule of $M$.

3.4. Lemma. If a submodule $N$ of an $R$-module $M$ satisfies the primeful property, then so do $\operatorname{rad} N$ and $S_{p}(N)$ for every $p \in V(N: M)$.

Proof. Suppose $p$ is a prime ideal of $R$ containing $(\operatorname{rad} N: M)$. Since $N$ satisfies the primeful property and $p \supseteq(N: M)$, there exists a prime submodule $P$ of $M$ containing $N$ such that $(P: M)=p$. It is clear that $P \supseteq \operatorname{rad} N$ and so $\operatorname{rad} N$ satisfies the primeful property. For the second part, let $p$ be a prime ideal of $R$ such that $p \supseteq\left(S_{p}(N)\right.$ : $M) \supseteq(N: M)$. Then there exists a prime submodule $P^{\prime}$ of $M$ containing $N$ such that 
$\left(P^{\prime}: M\right)=p$. Now, let $x \in S_{p}(N)$. There exists $s \in R \backslash p$ such that $s x \in N \subseteq P^{\prime}$. Therefore $x \in P^{\prime}$. Hence we have $S_{p}(N) \subseteq P^{\prime}$, as desired.

3.5. Theorem. Let $p$ be a prime ideal of $R$ and $N$ a submodule of an $R$-module $M$ satisfying the primeful property. Then the following statements are equivalent:

(i) $S_{p}(N)$ is a p-quasi-primary submodule of $M$;

(ii) $\left(S_{p}(N): M\right)$ is a p-quasi-primary ideal of $R$;

(iii) $\sqrt{\left(S_{p}(N): M\right)}=\left(\operatorname{rad} S_{p}(N): M\right)=p$;

(iv) $\left(S_{p}(N): M\right)$ is a p-primary ideal of $R$;

(v) $S_{p}(N)$ is a p-primary submodule of $M$.

Proof. (i) $\Rightarrow$ (ii) $\Rightarrow$ (iii) is clear by Lemma 3.4 .

(iii) $\Rightarrow$ (i). By [11, Result 1(1), page 2658], $S_{p}\left(S_{p}(N)\right)=S_{p}(N)$. It implies that $S_{p}\left(S_{p}(N)\right) \subseteq \operatorname{rad}\left(S_{p}(N)\right)$ and so $S_{p}(N)$ is a $p$-quasi-primary submodule of $M$ by Lemma 3.1 .

(iii) $\Leftrightarrow($ iv $) \Leftrightarrow(\mathrm{v})$ is obtained by [11, Theorem 2.3].

3.6. Corollary. Let $N$ be a p-quasi-primary submodule of an $R$-module $M$ satisfying the primeful property. Then the equivalent conditions in Theorem 3.5 hold.

Proof. Since $N$ satisfies the primeful property, Lemma 3.4 shows that

$$
p=\sqrt{(N: M)} \subseteq \sqrt{\left(S_{p}(N): M\right)}=\left(\operatorname{rad} S_{p}(N): M\right)
$$

On the other hand, Lemma 3.1 follows that $\left(S_{p}(N): M\right) \subseteq p$ and hence $p=\left(\operatorname{rad} S_{p}(N)\right.$ : $M)$. Thus (iii) of Theorem 3.5 holds.

3.7. Corollary. Let $N$ be a p-quasi-primary submodule of an $R$-module $M$ satisfying the primeful property. If $\left(S_{p}(N): M\right)$ is a radical ideal of $R$, then radN is a prime submodule of $M$.

Proof. It follows from Corollary 3.6 that $S_{p}(N)$ is a $p$-primary submodule of $M$ and so $S_{p}(N)$ is prime, since $\left(S_{p}(N): M\right)$ is a radical ideal of $R$. Now the proof is completed by Theorem 3.3.

3.8. Proposition. Let $N$ be a p-quasi-primary submodule of an $R$-module $M$ satisfying the primeful property. Then

$$
\sqrt{S_{p}(N: M)}=\sqrt{\left(S_{p}(N): M\right)}=p .
$$

In particular, $S_{p}(N: M)$ and $\left(S_{p}(N): M\right)$ are p-primary ideals of $R$.

Proof. Since $N$ is $p$-quasi-primary, Lemma 3.1 shows that $\left(S_{p}(N): M\right) \subseteq \sqrt{(N: M)}$. Thus we conclude that $(N: M) \subseteq S_{p}(N: M) \subseteq\left(S_{p}(N): M\right) \subseteq \sqrt{(N: M)}$, as required. The second part is clear.

3.9. Theorem. Let $N$ be a submodule of an $R$-module $M$ and $p$ a prime ideal of $R$ such that $p \subseteq(\operatorname{rad} N: M)$. Then the following statements are equivalent:

(i) $S_{p}(\operatorname{rad} N) \neq M$;

(ii) $(\operatorname{rad} N: M)=\left(S_{p}(\operatorname{rad} N): M\right)=p$;

(iii) $S_{p}(\operatorname{radN})$ is a p-prime submodule of $M$.

Further, if $N$ is a p-quasi-primary submodule of $M$, then the above statements are equivalent to:

(iv) $\operatorname{radN}$ is a p-prime submodule of $M$. 
Proof. (i) $\Rightarrow$ (ii). By replacing $N$ with $\operatorname{rad} N$ in [11, Theorem 2.1], we have $\left(S_{p}(\operatorname{rad} N)\right.$ : $M) \subseteq p$. Since $p M \subseteq \operatorname{rad} N$, we have $p \subseteq(\operatorname{rad} N: M) \subseteq S_{p}(\operatorname{rad} N: M) \subseteq\left(S_{p}(\operatorname{rad} N)\right.$ : $M) \subseteq p$, whence (ii) follows.

(ii) $\Rightarrow$ (iii). Using [11, Theorem 2.3] by replacing $N$ with $\operatorname{rad} N$.

(iii) $\Rightarrow$ (i) is clearly true.

(iii) $\Rightarrow$ (iv). Let $N$ be a $p$-quasi-primary submodule of $M$. It follows from (ii) and (iii) that $S_{p}(\operatorname{rad} N)$ is a $p$-prime submodule of $M$ where $(\operatorname{rad} N: M)=p$. It follows from Lemma $3.2, S_{p}(\operatorname{radN})=\operatorname{radN}$. Hence $\operatorname{radN}$ is a $p$-prime submodule of $M$.

$($ iv $) \Rightarrow($ iii $)$ is clear.

3.10. Proposition. Let $N$ be a quasi-primary submodule of an $R$-module $M$. If $p=$ $(N: M)$ is a prime ideal of $R$, then $S_{p}(N)=M$ or radN is a prime submodule of $M$.

Proof. Suppose $S_{p}(N) \neq M$. By [11, Proposition 2.4], $S_{p}(N)$ is a prime submodule of $M$. It follows from Lemma 3.2 that $\operatorname{radN}$ is a prime submodule of $M$.

3.11. Corollary. Let $N$ be a quasi-primary submodule of an $R$-module $M$ satisfying the primful property. If $p=(N: M)$ is a prime ideal of $R$, then rad $N$ is a prime submodule of $M$.

Proof. Since $N$ satisfies the primeful property, we have $\operatorname{rad} N \neq M$. Also, it follows from Lemma 3.1 that $S_{p}(N) \subseteq \operatorname{radN}$. Now Proposition 3.10 completes the proof.

3.12. Proposition. Let $N$ be a p-quasi-primary submodule of an $R$-module $M$ satisfying the primeful property. Then $\operatorname{rad}_{p}(N) \subseteq S_{p}(N+p M) \subseteq S_{p}(\operatorname{rad} N)$. In particular, $p=\left(\operatorname{rad} S_{p}(N): M\right)=\left(S_{p}(N+p M): M\right)$.

Proof. By [11, Theorem 4.3], $S_{p}(N+p M)$ is a $p$-prime submodule of $M$ and so $\operatorname{rad} S_{p}(N) \subseteq$ $S_{p}(N+p M)$. Suppose $x \in S_{p}(N+p M)$. Then $c x \in N+p M$ for some $c \in R \backslash p$. Since $\sqrt{(N: M)}=p$ and $c x \in \operatorname{radN}$, we conclude that $x \in S_{p}(\operatorname{rad} N)$. Also, we have $p=(\operatorname{rad} N: M) \subseteq\left(\operatorname{rad} S_{p}(N): M\right) \subseteq\left(S_{p}(N+p M): M\right)=p$, as required.

The following is a result of [11, Corollary 5.7] and Proposition 3.12.

3.13. Corollary. Let $m$ be a maximal ideal of $R$ and $N$ a m-quasi-primary submodule of an $R$-module $M$ satisfying the primeful property. Then $\operatorname{rad} N=\operatorname{rad} S_{m}(N)=$ $S_{m}(\operatorname{rad} N)=S_{m}(N+m M)$.

3.14. Theorem. Let $R$ be an Artinian ring and $M$ a module over $R$. If $N$ is a quasiprimary submodule of $M$ and $p \in V(N: M)$, then the followings hold.

(i) $\operatorname{radN}$ is a prime submodule of $M$.

(ii) $\operatorname{rad} S_{p}(N)=S_{p}(\operatorname{rad} N)=S_{p}(N+p M)$. In particular, $\operatorname{rad} S_{p}(N)$ is a prime submodule of $M$.

Proof. (i). Since $R$ is an Artinian ring, [2, Theorem 2.16] implies that $N$ satisfies the primeful property. Thus $(N: M)$ is a quasi-primary ideal of $R$. Since $R$ is zerodimensional, $\sqrt{(N: M)}=(P: M)$ for all prime submodules $P$ containing $N$. Hence $p=\sqrt{(N: M)}=(\operatorname{rad} N: M)$ is a prime ideal of $R$. Now if $\operatorname{rx} \in \operatorname{rad} N$ and $x \notin \operatorname{rad} N$, there is a prime submodule $P^{\prime}$ containing $N$ such that $r x \in P^{\prime}$ and $x \notin P^{\prime}$. Thus $r \in\left(P^{\prime}: M\right)=\sqrt{(N: M)}=(\operatorname{rad} N: M)$ and so $\operatorname{rad} N$ is prime.

(ii). Suppose $x \in S_{p}(N+p M)$. Then $c x \in N+p M$ for some $c \in R \backslash p$. Since $\sqrt{(N: M)}=p, c x \in \operatorname{rad} N$ and so $x \in S_{p}(\operatorname{rad} N)$. Thus $S_{p}(N+p M) \subseteq S_{p}(\operatorname{rad} N)$. Now if $x \in S_{p}(\operatorname{radN})$, there exists $c \in R \backslash p$ such that $c x \in P$ and so similar to the process of the proof (i), $x \in P$. Hence we have $x \in \operatorname{rad} S_{p}(N)$ and so $S_{p}(\operatorname{rad} N) \subseteq \operatorname{rad} S_{p}(N)$. 
Finally, by [12, Theorem 4.3], $S_{p}(N+p M)$ is a prime submodule of $M$ and hence $\operatorname{rad} S_{p}(N) \subseteq \operatorname{rad} S_{p}(N+p M)=S_{p}(N+p M)$.

\section{Torsioan and radical}

Recall that a torsion submodule of a module $M$ over a domain $R$, denoted by $T(M)$, is the submodule $\{x \in M: \operatorname{ann}(x) \neq 0\}$ of $M$. An $R$-module $M$ is said to be torsion(resp. torsion-free), if $T(M)=M$ (resp. $T(M)=0)$. Compare the following proposition with [8, Lemma 1].

4.1. Lemma. Let $M$ be an $R$-module. Let $N$ be a submodule of $M$ satisfying the primeful property. Then radN is a quasi-primary submodule of $M$ if and only if $(N: M)$ is a quasi-primary ideal of $R$ and $T(M / \operatorname{rad} N)=0$ as a $R / \sqrt{(N: M)}$-module. In this case radN is a prime submodule of $M$.

Proof. Suppose $\operatorname{radN}$ is a quasi-primary submodule of $M$. By Lemma 2.12 (i), $\sqrt{(N: M)}=$ $p$ is a prime ideal of $R$. If $x+\operatorname{rad} N \in T(M / \operatorname{rad} N))$, then $r x \in \operatorname{rad} N$, for some element $r \in R \backslash p$. Since $\operatorname{rad} N$ is $p$-quasi-primary, $x \in \operatorname{rad} N$ i.e. $T(M / \operatorname{rad} N)=0$. Conversely, $\sqrt{(N: M)}=p \neq R$ implies $\operatorname{rad} N \neq M$. If $r x \in \operatorname{rad} N$ and $r \notin p$, then $x+\operatorname{rad} N \in T(M / \operatorname{rad} N)$ and so $x \in \operatorname{rad} N$. Thus $\operatorname{rad} N$ is a quasi-primary submodule of $M$. In this case $\operatorname{radN}$ is prime by Lemma 2.12 .

4.2. Corollary. Let $N$ be a submodule of an $R$-module $M$ satisfying the primeful property. If $(N: M)$ is a quasi-primary ideal of $R$ and $T(M / \operatorname{rad} N)=0$ as a $R / \sqrt{(N: M)}$ module, then $N$ is a quasi-primary submodule of $M$.

Proof. The proof is clear by using Lemma 2.12 and Lemma 4.1.

4.3. Theorem. Let $R$ be a Dedekind domain and $N$ a submodule of an $R$-module $M$ satisfying the primeful property. The following are equivalent:

(i) $\operatorname{radN}$ is prime;

(ii) $M=\operatorname{rad} N \oplus N^{\prime}$ for some torsion-free submodule $N^{\prime}$ of $M$ or $(\operatorname{rad} N: M)=m$ for some maximal ideal $m$ of $R$.

Proof. $(i) \Rightarrow(i i)$. Suppose first that $\operatorname{radN}$ is a 0 -prime submodule of $M$. It follows from Lemma 4.1 that $M / \operatorname{rad} N$ is a torsion-free $R$-module. It follows from [5, Exercise 19.6(a)] that $M / \operatorname{rad} N$ is projective and hence $M=\operatorname{rad} N \oplus N^{\prime}$ for some submodule $N^{\prime}$. Clearly $N^{\prime}$ is torsion-free. Now, let $\operatorname{rad} N$ be a prime submodule of $M$ with $(\operatorname{rad} N: M) \neq 0$. Since $R$ is Dedekind domain, $(\operatorname{rad} N: M)$ is a maximal ideal of $R$, as desired.

(ii) $\Rightarrow(i)$. Assume that $M=\operatorname{rad} N \oplus N^{\prime}$ for some torsion-free submodule $N^{\prime}$ of $M$. Then $M / \operatorname{rad} N \simeq N^{\prime}$ follows that $M / \operatorname{rad} N$ is torsion-free and hence $\operatorname{rad} N$ is a 0 -prime submodule of $\mathrm{M}$ by [8, Lemma 1]. On the other hand, it is easy to verify that $\operatorname{radN}$ is prime when $(\operatorname{rad} N: M)$ is a maximal ideal.

4.4. Theorem. Let $R$ be a Noetherian domain and $M$ be a non-torsion $R$-module such that $T(M)$ is contained in only finitely many prime submodules of $M$. If $N$ is a quasiprimary submodule of $M$ satisfying the primeful property, then rad $N$ is prime.

Proof. We first assume that $(N: M)=0$. It follows from Corollary 3.11 that $\operatorname{rad} N$ is a prime submodule of $M$. Thus we may assume that $(N: M) \neq 0$. If $P$ is a prime submodule containing $N$, we have the chain $0=(T(M): M) \subset \sqrt{(N: M)} \subseteq(P: M)$ of prime ideals of $R$. If the later containment is proper, by [9, p.144] there are infinitely many prime ideals $p$ with $(N: M) \subset p \subset(P: M)$ and so we have infinitely prime submodules $P$ containing $T(M)$, a contradiction. Hence we have $\sqrt{(N: M)}=(P: M)$, 
for all prime submodules $P$ containing $N$. Now if $r x \in \operatorname{rad} N$ and $x \notin \operatorname{radN}$, there is a prime submodule $P$ containing $N$ such that $r x \in P$ and $x \notin P$ and therefore $r \in(P: M)=\sqrt{(N: M)}=(\operatorname{rad} N: M)$, as required.

For an $R$-module $M$ and $x \in M$, we mean that $(N: x)$ is the set $\{r \in R: r x \in N\}$. Now we have the elementary following lemma.

4.5. Lemma. Let $M$ be an R-module. Then $N$ is a quasi-primary submodule of $M$ if and only if $\sqrt{(N: M)}=\sqrt{(N: x)}$ for all $x \in M \backslash \operatorname{rad} N$.

In the following quasi-primary module is considered a module whose the zero submodule is quasi-primary.

4.6. Theorem. Let $M$ be a quasi-primary and primeful module over a one-dimensional domain $R$. Then either $\sqrt{\operatorname{ann}(M)}=0$ or $\sqrt{\text { ann }(M)}=\sqrt{(N: M)}$ for all proper submodules $N$ of $M$. In particular, if $M$ is a non-cyclic torsion module, then $\sqrt{(R x: M)}=$ $\sqrt{\operatorname{ann}(x)}$ for all $x \in M \backslash \operatorname{rad} 0$.

Proof. Suppose $\sqrt{\operatorname{ann(M)}} \neq 0$. Since $R$ is a one-dimensional domain, $\sqrt{\operatorname{ann}(M)}$ is a maximal ideal of $R$. It conclude that $\sqrt{\operatorname{ann}(M)}=\sqrt{(N: M)}$ for all proper submodules $N$. Since 0 is a quasi-primary submodule satisfying the primeful property, $\operatorname{rad} 0 \neq M$. Now if $M$ is a torsion module, then $\sqrt{\operatorname{ann}(M)} \neq 0$. Again since 0 is quasi-primary, $\sqrt{\operatorname{ann}(M)}=\sqrt{\operatorname{ann}(m)}$ for all $x \in M \backslash \operatorname{rad0}$ by Lemma 4.5. Since $R x$ is a proper submodule for all $x \in M$, by the first part $\sqrt{(R x: M)}=\sqrt{\operatorname{ann}(M)}=\sqrt{\operatorname{ann}(x)}$

4.7. Theorem. Let $M$ be a torsion module over a one-dimensional domain $R$. If $M$ is quasi-primary and primeful, then there exists a prime ideal $p$ of $R$ such that $r \notin p$ implies $r M=M$.

Proof. Suppose $p=\sqrt{\operatorname{ann}(M)}$. If $r M \neq M$, then by Theorem $4.6 r \in \sqrt{(r M: M)}=$ $\sqrt{\operatorname{ann}(M)}=p$.

4.8. Theorem. Let $M$ be a quasi-primary primeful and torsion module over a onedimensional domain $R$. If $p=\sqrt{\operatorname{ann}(M)}$ and $M_{p}$ is the localization of $M$ at $p$, then $M / S_{p}(0) \cong M_{p}$, an isomorphism of $R$-modules.

Proof. Consider the $R$-module homomorphism $\psi: M \longrightarrow M_{p}$, given by $m \mapsto m / 1$. To show that $\psi$ is an epimorphism, take any $m / s \in M_{p}$. Since $s \notin p, s M=M$ by Theorem 4.8 and so there exists $m^{\prime} \in M$ such that $m=s m^{\prime}$. Thus $m / s=s m^{\prime} / s=m^{\prime} / 1=\psi\left(m^{\prime}\right)$. Also it is easy to verified that the kernel of $\psi$ is $S_{p}(0)$. Hence $M / S_{p}(0) \cong M_{p}$.

\section{Acknowledgment}

The authors express their sincere gratitude to the editor and referee for the careful reading of the original manuscript and useful comments.

\section{References}

[1] Behboodi, M. On the prime radical and bear's lower nilradical of modules, Acta Math. Hungar., 3, 293-306, 2009.

[2] Behboodi, M., Aghasi, M. and Sabzevari, M. A structure sheaf on the spectrum of prime radical modules, J. Comm. Algebra., Accepted.

[3] Dauns, J. Prime submodules, Reine angew. Math., 298, 156-181, 1978.

[4] Ebrahimi Atani, S. and Yousefian Darani, A., On quasi-primary submodules, Chiang Mai J. Sci., 33 (3), 249 - 254, 2006.

[5] Eisenbud, D., Commutative algebra with a view toward algebraic geometry, (Springer-Verlag, 1994). 
[6] Fazaeli Moghimi, H. and Rashedi, F., Primary-like submodules satisfying the primeful property, Transactions on Algebra and its Applications, 1, 43-54, 2015.

[7] Fuchs, L. On Quasi-primary ideals, Acta Sci. Math. (Szeged), 11, 174-183, 1974.

[8] Jenkins, J. and Smith, P. F. On the prime radical of a module over a commutative Ring, Comm. Algebra, 20, 3593-3602, 1992.

[9] Kaplansky, I. Commutative rings, (The University of Chicago Press, 1974).

[10] Lu, C.P. A module whose prime spectrum has the surjective natural map, Houston J. Math., 33 (1), 125-143, 2007.

[11] Lu, C.P. Saturations of submodules, Comm. Algebra, 31 (6), 2655-2673, 2003.

[12] Lu, C.P. M-radical of submodules in modules II, Math. Japonica, 35 (5), 991-1001, 1990.

[13] McCasland, R. L. and Moore, M. E. Prime submodules, Comm. Algebra, 20 (6), 1803-1817, 1992.

[14] McCasland, R. L., Moore, M. E. and Smith, P. F. On the spectrum of a module over a commutative ring, Comm. in Algebra, 25 (1), 79-103, 1997.

[15] McCasland, R. L. and Smith, P. F. Generalised associated primes and radicals of submodules, Int. Electron. J. Algebra, 4, 159-176, 2008.

[16] Moore, M. E. and Smith, S. J. Prime and radical submodules of modules over commutative rings, Comm. Alegbra, 30, 5073-5064, 2002.

[17] Naghipour, R. and Sedghi, M. Weakly associated primes and primary decomposition of modules over commutative rings, Acta Math. Hungar. 110 (1-2), 1-12, 2006.

[18] Sharif, H., Sharifi, Y., and Namazi, S. Rings satisfying the radical formula, Acta Math. Hungar., 71(1-2), 103-108, 1996.

[19] Smith, P. F. Primary modules over commutative rings, Comm. Algebra, 43 (2001), 103-111.

[20] Pusat-Yilmaz, D. and Smith, P. F. Radicals of submodules of free modules, Comm. Algebra, 27 (5), 2253-2266, 1999.

[21] Pusat-Yilmaz, D. and Smith, P. F. Modules Which Satisfy the Radical Formula, Acta Math. Hungar., 95 (1-2), 155-167, 2002. 
